\section{WILD IMMUNOLOGY}

\section{A more natural response}

PLoS Biol. https://doi.org/10.1371/journal.

pbio.2004108 (2018)

Mouse experiments are conventionally done with tightly controlled environmental and microbiological parameters; however, this probably does not capture the entire complexity of host-pathogen interactions. In PLoS Biology, Graham and colleagues try to address this shortfall in understanding by using conventional C57BL/6 mice kept in the laboratory together with those in semi-natural outdoor conditions (protected from predation but otherwise fully exposed to the environment). The 'wild' mice show greater diversity of the microbiota and, when experimentally infected with Trichuris muris, develop a greater burden of this nematode. Uninfected 'wild' mice have elevated baseline type 2 immune responses but, unexpectedly, develop a more robust type 1 immune response after being infected with T. muris. This type 1 skewing is inefficient in clearing nematode infections and probably contributes, at least in part, to the greater nematode parasite burden. $\quad Z F$

https://doi.org/10.1038/s41590-018-0122-2

\section{MACROPHAGES}

\section{Immunoregulatory itaconate}

Nature https://doi.org/10.1038/s41586-018-

0052-z (2018)

Itaconate is a metabolite that arises from the tricarboxylic acid cycle. In Nature, Artyomov and colleagues reveal anti-inflammatory roles for itaconate in macrophages. Itaconate, or its membrane-permeable derivative dimethyl itaconate (DI), induces electrophilic stress in cells by decreasing the effective concentration of intracellular glutathione, thereby decreasing cellular redox buffering capacity. Itaconate and DI activate the integrated stress-response pathway that includes upregulation of the translational regulator ATF3. This scenario suppresses expression of the cytokines IL- 6 and IL- 12 by interfering with the translation of transcripts encoding $\mathrm{I} \kappa \mathrm{B} \zeta$, a regulator of genes encoding secondary pro-inflammatory response molecules. Notably, in a mouse model of psoriasis, treatment with DI blunts IL-17-stimulated pro-inflammatory responses in keratinocytes and diminishes psoriatic skin lesions. LAD

https://doi.org/10.1038/s41590-018-0123-1

\section{B CELLS \\ IgD's role in vivo \\ elife 7, e35074 (2018)}

Mature naive B cells express surface IgM and IgD as BCRs, yet the distinct role of IgD has remained unknown. In eLife, Zikherman and colleagues show that IgD increases the dynamic range of BCR signaling in vivo but restrains the generation of antibodies by autoreactive B cells. They use reporter mice with IgM-only or IgD-only B cells that express a Nur77-eGFP reporter as a 'readout' of BCR signaling in vivo. IgM-only B cells show stronger signaling than that of IgDonly B cells in response to endogenous self antigen. Such self-reactive IgM-only B cells differentiate into short-lived plasma cells, whereas IgD-only B cells are less sensitive to antigen despite expressing more surface BCR molecules per cell. This difference in sensitivity might explain why wild-type $\operatorname{IgM}^{\mathrm{lo}} \operatorname{IgD}{ }^{\text {hi }} \mathrm{B}$ cells can capture endogenous antigen but fail to spontaneously become autoantibody-secreting cells.
MICROGLIA

\section{Training and tolerance}

Epigenetically mediated immunological imprinting can manifest as training or tolerance, which enhance inflammation or suppress it, respectively. In Nature, Neher and colleagues show that one intraperitoneal injection of LPS $(1 \times$ LPS $)$ induces training, while treatment with four injections of LPS ( $4 \times$ LPS) induces tolerance in brain microglia, manifested by the respective increased or decreased induction of the inflammatory cytokines TNF, IL-1 $\beta$ and IL- 6 in the brain. In APP2 mice, a model of Alzheimer's disease, or in mice with induced ischemia, 1×LPS increases neuropathology, while $4 \times$ LPS decreases it. Training (1×LPS) induces the amplification of inflammation-related pathways, such as HIF-1 $\alpha$-dependent signaling and a metabolic switch to glycolysis, that are already upregulated in APP2 mice relative to their activity in wild-type mice, while tolerance $(4 \times$ LPS) downregulates these pathways. The effect of other inflammatory stimuli remains to be tested.

\section{MECHANOBIOLOGY}

\section{Flowing mechanics}

Nat. Meth. https://doi.org/10.1038/nmeth.4639 (2018)

Analysis of cellular mechanical properties has shed light on many fundamental biological processes, such as mitosis and $\mathrm{T}$ cell activation. Typically, such analysis is performed using atomic-force microscopy, a laborious and lowthroughput process (tens of cells per hour) that also lacks molecular specificity. In Nature Methods, Guck and colleagues report their development of a fluidics platform that essentially combines the properties of fluorescence flow cytometry with cell-mechanical analysis. This approach allows vastly higher throughput (up to 100 cells per second) than that of atomic-force microscopy but can also provide one-dimensional fluorescent information, such as that pertaining to cell-subset identity. They validate the platform using various approaches; for example, they investigate the mechanics of progenitors of human hematopoietic stem cells gated into CD $34^{+}$or CD $34^{-}$ populations. In principle, this approach might eventually allow label-free sorting of cells on the basis of mechanical properties alone.

https://doi.org/10.1038/s41590-018-0125-z

\section{ANTIVIRAL RESPONSES}

\section{Mn potentiates cGAS Immunity 48, 675-678 (2018)}

Manganese ( $\mathrm{Mn}$ ) regulates the function of various types of enzymes. In Immunity, Jiang and colleagues show that $\mathrm{Mn}^{2+}$ is required for the induction of interferon responses to DNA viruses. Viral infection induces the cytoplasmic release of $\mathrm{Mn}$ from the Golgi and mitochondria. $\mathrm{Mn}^{2+}$ increases the enzymatic activity of the DNA sensor cGAS and its sensitivity to double-stranded DNA, as well as the affinity with which the signaling intermediate cGAMP binds to the downstream adaptor STING. Mice on a Mn-deficient diet are highly susceptible to infection with sub-lethal doses of the viruses HSV and VACV, with alveolar and peritoneal macrophages from these mice showing diminished type 1 interferon responses to DNA viruses but not to RNA viruses.

https://doi.org/10.1038/s41590-018-0126-y

Laurie A. Dempsey, Zoltan Fehervari and loana Visan 